\title{
CULTURAL HISTORICAL ACTIVITY THEORY, DOUBLE STIMULATION, AND CONFLICTS OF MOTIVES IN EDUCATION SCIENCE: WHERE HAVE WE BEEN? (2012-2021)
}

Guillaume Isaac

guillaume.isaac@univ-cotedazur.fr

PhD candidate

Laboratoire d'Innovation et Numérique pour l'Education (LINE)

Université Côte d'Azur

Sylvie Barma

sylvie.barma@fse.ulaval.ca

Full Professor

Faculté des Sciences de l'Éducation

Université Laval

Margarida Romero

margarida.romero@unice.fr

Full Professor

Laboratoire d'Innovation et Numérique pour l'Éducation

Université Côte d'Azur

\begin{abstract}
Cultural Historical Activity Theory (CHAT) can be used both as a theoretical and an analytical framework. It has been used in a wide variety of contexts over a wide variety of research objects in education and other fields requiring a comprehensive understanding of interactions within a system. At a theoretical level, the relation between double stimulation and instrumental mediation has been studied and understood, yet the link between double stimulation and conflicts of motives is understudied. How has scientific literature considered this avenue for research over the past ten years? A systematic literature review was conducted, and thematic analysis was performed in order to identify themes. As of the first quarter of 2021, scientific literature in education sciences does not yet reflect the interest and potential of this research avenue. Double stimulation and its relation to conflicts of motives are key components to form volitional action in education contexts and is therefore a valuable research object.
\end{abstract}

Keywords : Cultural Historical Activity Theory, CHAT, Double Stimulation, Conflicts of motives 


\section{Résumé}

La théorie historico-culturelle de l'activité peut être utilisée en recherche comme cadre théorique analytique. En tant que telle, c'est un cadre qui été utilisé dans une grande variété de contextes et d'objets de recherche en éducation et dans d'autres champs qui requièrent une compréhension étendue des interactions dans un système. Si d'un point de vue théorique, la relation entre stimulation duale et médiation instrumentale a été étudiée et comprise, il n'en est pas de même pour ce qui concerne le lien entre stimulation duale et conflit de motifs. Dans quelle mesure la littérature scientifique a-t-elle pris en compte cette piste de recherche ces dix dernières années ? Pour tenter de répondre à cette question, une revue systématique de littérature et une analyse thématique ont été menées afin d'identifier les thèmes pertinents. Les résultats de cette étude montrent qu'à compter du premier trimestre 2021, la littérature scientifique en sciences de l'éducation ne reflète l'intérêt et le potentiel de recherche identifiés ailleurs. La relation entre stimulation duale et conflits de motifs sont des éléments clés de la genèse de la volition et de l'action en contextes éducatifs; ces deux concepts forment donc un objet de recherche pertinent.

Mots-clés : Théorie Historico-culturelle de l'Activité, Stimulation duale, CHAT, conflits de motifs

\section{Note d'auteur}

Sylvie Barma, identifiant ORCID : 0000-0002-3603-2552

Margarida Romero, identifiant ORCID : 0000-0003-3356-8121

All correspondence concerning this article should be addressed to Guillaume Isaac, Laboratoire d'Innovation et Numérique pour l'Enseignement (LINE), INSPE Liégeard, Université Côte d'Azur, 43 Avenue Stephen Liégeard 06100 Nice, guillaume.isaac@univcotedazur.fr 
Revue internationale du CRIRES: innover dans la tradition de Vygostky (2021) ISSN 2291-6717, vol5, no2, 86-94

Cultural historical activity theory, double stimulation, and conflicts of motives in education science: Where have we been? (20122021)

\section{Introduction}

The rationale for writing this article came from a twofold observation formulated over two scientific papers. In a context of continuous changes, understanding the formation of will in contexts of uncertainty is essential as far as educational challenges are concerned, as the formation of will is at the very core of transformative agency (Sannino \& Laitinen, 2015). Agency has been the subject of a number of studies through double stimulation, and yet somewhat surprisingly the link between volition and double stimulation remains understudied (Sannino, 2015). Sannino (2015) further suggested that even though instrumental mediation had been a widely researched topic, the widening of focus over double stimulation was called for; namely, the study of the formation of stimuli, particularly through the study of conflicts of motives was subsequently identified as a valuable avenue for research. This study seeks to identify the main topics of research on double stimulation in education research and psychology over the past ten years (2012-2021) to determine whether Sannino's call has been answered.

\section{Definition of the problem}

The problem addressed by this review originates from the fact that double stimulation appears in a very fragmented way in the literature (Sannino \& Laitinen, 2015) despite being among the foundational principles of a deep tradition of formative interventions, from Vygotsky to Change Laboratories in the third generation of Cultural Historical Activity Theory (CHAT). Ploettner and Tresseras (2016) report the point of view according to which Engeström argues that activity theory should not be reduced to technical mediation and double stimulation should be used to investigate deeper the reasons activities are conducted, their origins and their connections to society. This, according to the author, is among the main differences between CHAT and other theoretical frameworks such as situated action or sociocultural studies. Therefore, we sought to identify the type of research, the public, the themes that were explored, the type of results, the kind of methods employed, the prevalence of the CHAT theoretical framework as far as double stimulation is concerned. How has the notion been harnessed as a research avenue, and how -if at all- has it penetrated the field of education at large?

\section{Methodology}

As stated above, the main research questions asked in this study is the following: (1) How has double stimulation been used in education science research over the past ten years? To answer the question a qualitative literature review was conducted in the form of a textual narrative synthesis to identify all relevant information to the use of double stimulation in education in a selection of scientific articles detailed in the following section (Barnett-Page \& Thomas, 2009).

The research was conducted over a sample of papers written in English language to ensure the broadest possible view over topics and methods used. The research strategy used was "kw:("activity theory") OR kw:("cultural historical activity theory") AND kw:("double stimulation") AND (eu:Peerreviewed)". The research was performed on a sample of peer-reviewed articles exclusively over a period of ten years (2012-2021). Part of the reason for this timeframe was to interrogate a bracket in time prior and posterior to Sannino (2015) and evaluate whether the research avenues suggested in the paper had been pursued or not.

The results were retrieved from ERIC and PsyArticles databases on 21/03/21 at 11:00am European time using the Sofia search engine service provided by the Université Laval Library and 
Revue internationale du CRIRES: innover dans la tradition de Vygostky (2021) ISSN 2291-6717, vol5, no2, 86-94

Cultural historical activity theory, double stimulation, and conflicts of motives in education science: Where have we been? (20122021)

returned 23 results. ERIC database was chosen due to its large collection of articles and academic resources specialized on educational topics. Since the research is being made and retrieved in English language, ERIC was identified as the most comprehensive source. The PsycArticles database was selected as a complementary choice since it is the main outlet for the American Psychology Association and comprises resources by the American Psychological Association, the APA Educational Publishing Foundation, the Canadian Psychological Association, and Hogrefe \& Huber. All activity theory generations being affiliated with the discipline of psychology, it was therefore decided to complete a comprehensive search.

The initial batch of articles was consequently treated through a first round of superficial analysis to suppress duplicate entries and ensure that peer-reviewed scientific papers were exclusively kept within the corpus. As stated above, the object of the research was initially circumvented to education, and later extended to organization fields and psychology. Further, interviews, editorials, book chapters and methodology reviews were excluded to focus exclusively on studies presenting data and a structured method of analysis. A quick word search for the key terms "double stimulation" within each article was also performed at this stage and all articles not using them as a concept or as a method of analysis were excluded. The corpus presented in this review was consequently shrunk to the somewhat surprisingly low number of 6 references, the results of which are commented below.

The analysis was further refined through a second round through the following categories: (a) theme and field of study, (b) particular use of the concept of double stimulation and (c) type of results obtained. The reason for this choice of categories was to identify main avenues for research for and about double stimulation.

The limits of this method are mainly the small number of articles treated, the subjectivity inherent to the use of narrative methods of analysis in literature reviews, which could have been dampened using thematic synthesis, meta-analysis, or grounded theory methodology. As stated initially, the purpose of this review was rather to identify gaps in the literature and identify whether Sannino's (2015) promising step forward had been followed up or not. Nonetheless, the subjective nature of the methodology involved, coupled to the inductive process of analysis of the data and to the fact that this review was performed without a quality assessment process are its main limits.

\section{Results}

The corpus commented below is made of seven references spanning a ten-year period from 2012 (Virkkunen \& Ristimäki, 2012) to 2021 (Morselli \& Sannino, 2021). It must be observed that the initial impetus for this review rests mostly on Sannino (2015), her influence on this research area remains strong as the research query returned two more articles signed or co-signed by her, therefore leaving a noticeable mark on the overall direction of this review.

As far as themes and fields of studies are concerned, the articles retained fall into two broad categories: either they deal with professional transformation (Virkkunen \& Ristimäki, 2012; Penuel, 2014), whether through the conduct of change laboratories as the main methodologies or not, or they deal with dynamics of actors in interaction with a set of artefacts and/or persons (Barma et al, 2015; Kucirkova et al., 2015; Thompson, 2015; Sannino, 2016; Morselli \& Sannino, 2021). Half of the papers presented deal directly with formative interventions (Virkkunen \& Ristimäki, 2012; Thompson, 2015; Morselli \& Sannino, 2021) making it at the forefront of double stimulation use both for research purposes and professional development. One paper among those three (Morselli \& Sannino, 2021) reports a "pure" Change Laboratory in the tradition of Engeström (1987) while the other two somewhat tailored the initial methodologies to suit their own needs: 
Revue internationale du CRIRES: innover dans la tradition de Vygostky (2021) ISSN 2291-6717, vol5, no2, 86-94

Cultural historical activity theory, double stimulation, and conflicts of motives in education science: Where have we been? (20122021)

study of the formation of concepts as transitional artefact in professional settings on the one hand (Virkkunen \& Ristimäki, 2012) and intersection of design research and change laboratories (Penuel, 2014) on the other hand. The cited papers dealt mostly with educational settings or environments apart from Virkkunen and Ristimäki (2012); it should also be noted that, although the writings of Sannino $(2015,2016)$ have multiple applications and prevalence in the field of education, the experiment of the waiting room for collectives hails more to psychology research than education science in the strictest sense.

According to Kucirkova (2015), the idea of double stimulation initially stemmed from the metaphor of dual representation according to which an object can be thought of on two planes: a concrete level as a physical object and a symbolic one charged with sense and meaning. By investigating concrete and symbolic representations, one gains the ability to investigate both reasoning abilities and symbolic understanding through specific cultural tools.

Vygotsky sought to investigate the development of knowledge through different experimental iterations of double stimulation. Kucirkova (2015) mentions the wooden blocks experiment, which allowed the researchers to investigate how children develop their knowledge through conversing with an adult; it allowed the researcher to witness first-hand the development of reasoning skills through handling of blocks and conversation with the children participating in the experiment. It also allowed for understanding of investigating the influence of prompting conceptual understanding. In this sense, double stimulation makes the development of new knowledge visible.

A second experiment lead by Vygotsky to refine his understanding of Double Stimulation is what is often referred to as "the meaningless situation" or the "waiting experiment" as reported by Morselli and Sannino (2021); it consists of leaving a participant unattended in an empty room without instructions. The results in various settings show that participants make decisions to escape the situation based on external objects (in this setting a clock) and generate an act of will relative to the position of the hands on the clock to ease the conflict caused by two distinct motives: the motive of waiting and the motive of leaving. It is what Vygotsky refers to as "first stimulus" whereas the clock acquires the function of second stimulus and allows for restoring equilibrium in the subject's psyche. According to Morselli and Sannino (2021) and echoing Kucirkova's (2015) interpretation of the book, the clock thus gains new meaning and becomes a purposeful sign on which the subject builds intention or will. They also mention several famous examples in the literature such as tying a knot as a reminder to stimulate memory which allows people to create temporary links and "make sense" of stimuli.

Thompson (2015) mainly uses Double Stimulation as a methodological tool; he elaborates on Kucirkova's line of thinking in the context of formative interventions as far as the analysis of interaction and development are concerned. Thompson interprets the aim of Double Stimulation as the result of Vygotsky's quest to understand interaction and development by focusing the researcher on dynamic and historical channels of transformation. The main purpose of this theoretical endeavor was to observe and understand the development and inner structure of higher mental processes. According to Thompson, Vygotsky meant to prove that all human action is defined by the ways we use culturally and socially acquired knowledge to make sense of the world.

In this respect, the principle of double stimulation is initially described as a situation that confronts an individual or group of individuals with a problem or dilemma which they do not know yet how to solve. Thompson (2015) identifies after Vygotsky the initial situation as the first stimulus or "stimulus-end", and it constitutes the object of the activity; the second stimulus (or "stimulus-means" or "auxiliary means") represents the potential means of solving the dilemma in 
Revue internationale du CRIRES: innover dans la tradition de Vygostky (2021) ISSN 2291-6717, vol5, no2, 86-94

Cultural historical activity theory, double stimulation, and conflicts of motives in education science: Where have we been? (20122021)

the form of tools or artefacts. Following Sannino (2015), the process of double stimulation is initiated with a conflict of motives which is resolved through volitional action or will.

Morselli and Sannino (2021) describe the decision-forming apparatus further and elaborate on double stimulation. To them, the decision-forming process is formed by two apparatuses; in the first, the process of decision to behave in a certain way through an auxiliary motive is set, and Apparatus 2 allows for implementation of the decision. Apparatus 1 comprises four distinct phases, the first one of which is the confrontation to two antagonistic stimuli; in phase 2 a conflict of motives is engaged because of the antagonistic stimuli. The third phase corresponds to the selection of a stimulus and its conversion to an auxiliary motive. Phase 4 picks an occurrence of the auxiliary motive and attaches the chosen response to it. At this point in the process, apparatus 2 then follows suit as essentially an execution device, as if a response to a stimulus, in what is mostly described as an automatic mode of action.

The account of the first two initial experiments used to refine the model of double stimulation provide insight into its genealogy; it was initially used as an experimental method to gain access to otherwise inaccessible mental functions. Since its origins, however, the uses of double stimulation have varied significantly: Sannino (2015) establishes double stimulation as a "method", a "process", a "theory-method" and a "principle". Ploetter and Tresseras (2016) report Engeström's statement about the importance of using Double Stimulation not only as "a method understood as a technique. It's a Method (with the capital letter) understood dialectically as a generative principle that will be instrumental to develop our theory further" (p.97).

Sannino, in the same article, argues the case further: double stimulation is rooted in a long tradition in Soviet psychology, which are the origin of what is nowadays called formative experiments (Ploettner \& Tresseras, 2016). Its main principle is faithful to both examples reported above: offering a set of two stimuli, the second one being an artefact or a model to engage participants in redesigning their activity. As such, a variety of objects have been studied over time through the prism of double stimulation.

Virkkunen and Ristimäki (2012) have used double stimulation for its potential of expansive reconceptualization of activity and as such provides a good example of the particular use of double stimulation within a given context; the process involved was tailored to their needs and is described as follows: a first stimulus representing the need for a new strategy, the available concepts used as second stimuli, and finally a solution and its consequences. In the same vein and expanding on the initial model of Double Stimulation, Penuel (2014) demonstrates three forms allowing for transforming activities: (a) articulation is the process by which an activity recovers sense and coherence that it had lost; (b) cross-appropriation allows for tools or ideas to be adapted from other activities; (c) reconfiguration allows for a marginal aspect of an activity to become dominant, thus transforming the whole pattern. Both examples echo what Morselli and Sannino (2021) describe as the power to understand how individuals and collectives generate will in circumstances of cognitive conflict and ambiguity.

To elaborate on what the differences are with other typical methods of research in education, Engeström (2009) lists three main crucial points of divergence from linear interventions on design experiments. According to him, in linear interventions contents and goals are known in advance by the researchers who aim for an object that is at least known in some form to them. Formative interventions on the other hand encourage participants to construct and come up with solutions which are not known by researchers beforehand. The second difference is that the content of formative interventions, as well as its course are negotiated rather than expected. Finally, although linear interventions foster control of the variables and achieve standard results in analog 
Revue internationale du CRIRES: innover dans la tradition de Vygostky (2021) ISSN 2291-6717, vol5, no2, 86-94

Cultural historical activity theory, double stimulation, and conflicts of motives in education science: Where have we been? (20122021)

settings, formative interventions seek to generate concepts and solutions that can be used in other settings to foster appropriate new solutions.

Sannino (2016) recognizes the potential of double stimulation to study human behavior taking uncertainty into account and breaking away from methodologies ruled by control and predictability. At an epistemological level, she puts forward the idea according to which the social and political implications of research endeavors impose the ability to deal with and account for uncertainty and asserts the necessity to consider the input of participants which are usually neglected in experimental settings in psychology. Under the prism of double stimulation, situations of uncertainty and cognitive incongruity bring human beings facing conflicts of motives to rely on artifacts which serve the function of auxiliary motives, and which help them undertake volitional actions.

As stated above, double stimulation is therefore a promising way of understanding how subjects transform their condition (Sannino, 2015). The two apparatuses of decision forming taken from Sannino $(2015,2016)$ state the importance of conflicts of motives which happen as a result of the collision between opposite inclinations as is the case in situations of ambiguity or uncertainty.

According to Morselli and Sannino (2021), conflicts of motives in particular are the triggers of acts of will that allow the subject to take control of their environment and are therefore an important element of double stimulation. In Barma et al. (2015), double stimulation is triggered by the expansive resolution of conflicting motives experienced by two teachers leading them to engage in transformative agency. Struggles, obstacles, clashes and tensions are at the core of the first stimulus leading to a decision forming process giving voice to a new pedagogical strategy in the form of a second stimulus transforming the conditions of the practitionners.

Engeström identifies four types of tensions recurring in data generated through Change Laboratories: dilemmas, conflicts, conflicts of motives, double binds (Ploettner \& Tresseras, 2016). According to him, human beings tend to think dilemmatically by contrasting opposing elements and is therefore a natural base for generating conflicts, each type displaying more transformative energy. As such, conflicts represent the step when participants' views clash among one another according to the context. Critical conflicts emerge when participants are paralyzed; as such, critical conflicts have the power to lead them to understand what the forces are that make action so hard to undertake. Critical conflicts, which Sannino and Laitinen also refer to as conflicts of motives is a clash between opposite motives (Sannino \& Laitinen, 2015); it is a sign that a subject's will is split between different courses of actions, and therefore unable to act. This concept and its study in CHAT has led to understanding and formalizing the notion of transformative agency which is at play in much of Change Laboratories activities. Finally, Engeström identifies the category of double binds, which bridge the gap between individual and collective dimensions and which trigger action by federating forces to overcome a crisis (Ploettner \& Tresseras, 2016).

Plotter and Tresseras (2016) report further on Engestöm and Sannino's thoughts according to which although the idea of Double Stimulation stems from Vygotsky, it is rooted in a long tradition in Soviet psychology of formative experiments, which have served as model for the Change Laboratory. Morselli and Sannino (2021) expand on this idea to the use of formative interventions in educational contexts; according to them, double stimulation is particularly useful in contexts of teacher training or school development programs, when a community of actors are faced with problems that affect them in significant ways. Double stimulation offers a way to address the problem while offering to take concrete actions to make a difference. 
Revue internationale du CRIRES: innover dans la tradition de Vygostky (2021) ISSN 2291-6717, vol5, no2, 86-94

Cultural historical activity theory, double stimulation, and conflicts of motives in education science: Where have we been? (20122021)

Morselli and Sannino (2021) get into further details describing the Change Laboratory methodology arguing that its premise is to organize a space with a rich kit of instruments to reflect and model new iterations of work activity. Change laboratories have been widely used in a variety of organizations both in the public and the private sector. Morselli and Sannino state after Engeström that the Change Laboratory format ideally involves 15 to 20 participants over a cycle of 10 sessions organized around weekly meetups the frequency of which stretches over time; the process is systematically overseen by researchers to analyze and understand the problems and contradictions experienced by participants and to support the process of what they define as concept formation, also conceptualized by Virkkunen and Ristimäki (2012). A rigorous application of double stimulation to a setting such as the change laboratory locates the first stimulus as a mirror in which a problem is presented by means of emerging data such as video. The second stimulus is then articulated by researchers who bring conceptual tools to be used as instruments for analyzing the initial situation and build an adequate response collectively according to contents that are relevant to their practices and cultures and use them as starting points to evolve progressively. Change laboratories additionally provide analysis and data of each session to help with the identification of stimuli.

The use of double stimulation in the context of Change Laboratories to bring change to collective activities is further commented on by Morselli and Sannino (2021) who assert the centrality of agency as the main purpose and goal of such a process, and ultimately as one of the main reasons to use double stimulation in the first place. The authors state that double stimulation has helped to better understand the principle of agency as an experience as well as a theoretical construct. In this respect, the model described by Sannino (2015, 2016) allows for testing on individuals and collectives, which is of particular importance to the present study.

In 2009, Engeström identified further work on double stimulation as a potential means to develop activity-theoretical studies, namely living movement, breaking away, double stimulation, stabilization, and boundary crossing. Later Sannino (2015) established double stimulation as a primary axis of research, with a particular emphasis on conflicts of motives in the generation of individual and collective will. This review did not identify this line of research as a trend in double stimulation under a CHAT perspective, with the exception of Sannino expanding her own work, and therefore establishes it as a worthy research endeavor. A complimentary search was performed to confirm the fact that conflicts of motives have been an understudied topic over the last ten years with only two results to the query.

\section{Conclusion}

To echo Sannino (2015), a deeper analysis of the process of double stimulation relating to its dialectical relation to conflicts, contradictions and double binds still appears relevant. This review shows that despite limitations integral to its methodology, the initial question about the liveliness of research on conflicts of motives and double stimulation reveals a gap about the use of double stimulation in student-centered contexts of learning. As stated in the introduction, understanding the volitional process is crucial to unveil learning processes related to agency and therefore has implications at institutional level as well as for classroom management (Sannino, 2015). Indeed, the learning processes made accessible by double stimulation are of particular importance to the understanding of collective dynamics of learning and negotiation, such as collaborative and creative problem solving. Generally speaking, understanding the management of conflicts opens the understanding of cross-disciplinary negotiations, sharing of expertise and experiences, the process of learning from one another, building a common language and common decision-making (Zahedi et al., 2017). Additional effort is therefore required in the future to fill 
Revue internationale du CRIRES: innover dans la tradition de Vygostky (2021) ISSN 2291-6717, vol5, no2, 86-94

Cultural historical activity theory, double stimulation, and conflicts of motives in education science: Where have we been? (20122021)

the gap of knowledge on psychological processes involved, and within an extended timeframe extending beyond the thirty minutes of the waiting experiment (Sannino, 2016).

\section{References}

Barma, S., Lacasse, M., \& Massé-Morneau, J. (2015). Engaging discussion about climate change in a Quebec secondary school: A challenge for science teachers. Learning, Culture and Social Interaction, 4, 28-36.

Barnett-Page, E., \& Thomas, J. (2009). Methods for the synthesis of qualitative research : A critical review. BMC Medical Research Methodology, 9(1), 59. https://doi.org/10.1186/1471-2288-9-59

Engeström, Y. (1987). Learning by Expanding. Cambridge University Press.

Engeström, Y. (2009). The future of activity theory: A rough draft. Learning and expanding with activity theory, 303-328.

Kucirkova, N., Sheehy, K., \& Messer, D. (2015). A Vygotskian perspective on parent-child talk during iPad story sharing: PARENT-CHILD TALK DURING iPAD STORYSHARING. Journal of Research in Reading, 38(4), 428-441. https://doi.org/10.1111/1467-9817.12030

Morselli, D., \& Sannino, A. (2021). Testing the model of double stimulation in a Change Laboratory. Teaching and Teacher Education, 97, 103224. https://doi.org/10.1016/j.tate.2020.103224

Penuel, W. R. (2014). Emerging Forms of Formative Intervention Research in Education. $\begin{array}{llll}\text { Mind, Culture, and } & \text { Activity, } 117 .\end{array}$ https://doi.org/10.1080/10749039.2014.884137

Ploettner, J., \& Tresseras, E. (2016). An interview with Yrjö Engeström and Annalisa Sannino on activity theory. Bellaterra Journal of Teaching \& Learning Language \& Literature, 9(4), 87. https://doi.org/10.5565/rev/jt13.709

Sannino, A. (2015). The principle of double stimulation : A path to volitional action. Learning, Culture and Social Interaction, 6, 1-15. https://doi.org/10.1016/j.lcsi.2015.01.001

Sannino, A. (2016). Double Stimulation in the Waiting Experiment with Collectives : Testing a Vygotskian Model of the Emergence of Volitional Action. Integrative Psychological and Behavioral Science, 50(1), 142-173. https://doi.org/10.1007/s12124-015-9324-4

Sannino, A., \& Laitinen, A. (2015). Double stimulation in the waiting experiment: Testing a Vygotskian model of the emergence of volitional action. Learning, Culture and Social Interaction, 4, 4-18. https://doi.org/10.1016/j.lcsi.2014.07.002

Thompson, I. (2015). Researching contradictions : Cultural historical activity theory research (CHAT) in the English classroom. English in Australia, 50(3), 21-26. WorldCat.org. Virkkunen, J., \& Ristimäki, P. (2012). Double Stimulation in Strategic Concept Formation: An Activity-Theoretical Analysis of Business Planning in a Small Technology Firm. Mind, Culture, and Activity, 19(3), 273-286. https://doi.org/10.1080/10749039.2012.688234

Zahedi, M., Tessier, V., \& Hawey, D. (2017). Understanding Collaborative Design Through Activity Theory. The Design Journal, 20(sup1), S4611-S4620. https://doi.org/10.1080/14606925.2017.1352958 